\title{
Influence of mitochondrial DNA 5178 C/A polymorphism on serum cholesterol changes: a short-term follow-up in middle-aged Japanese men
}

\author{
Rie Honmyo $\cdot$ Akatsuki Kokaze $\cdot$ Kanae Karita • \\ Masao Yoshida • Mamoru Ishikawa • \\ Hideki Ohno
}

Received: 18 February 2011 / Accepted: 12 January 2012/Published online: 19 February 2012

(C) The Japanese Society for Hygiene 2012

\begin{abstract}
Objective The mitochondrial DNA 5178 cytosine/ adenine (Mt5178 C/A) polymorphism is reportedly associated with longevity in the Japanese population, and the Mt5178A genotype may exert anti-atherogenic effects. The aim of this study was to determine whether there were longitudinal differences in serum lipid levels between carriers of the Mt5178C genotype and those of the Mt5178A genotype and to assess the impact of these genotypes on serum cholesterol levels.

Methods The serum levels of total cholesterol (TC), highdensity lipoprotein cholesterol (HDLC), and low-density lipoprotein cholesterol (LDLC) were measured and the Mt5178 C/A genotypes determined in 110 Japanese men aged 41-66 (mean 52.3) years who had received medical checkups twice in the period 1999-2005. The longitudinal changes of TC, HDLC, and LDLC were calculated according to genotype.

Results The serum levels of TC at baseline and follow-up were significantly different, whereas those of HDLC and LDLC were not. The changes in HDLC differed significantly between the two Mt5178 C/A genotype groups, with the changes in HDLC level being significantly greater in
\end{abstract}

R. Honmyo · K. Karita ( () - M. Yoshida - M. Ishikawa •

H. Ohno

Department of Public Health, Kyorin University School

of Medicine, 6-20-2 Shinkawa, Mitaka,

Tokyo 181-8611, Japan

e-mail: Kanae@ks.kyorin-u.ac.jp

\section{A. Kokaze}

Department of Public Health, Showa University School

of Medicine, Shinagawa-ku, Tokyo 142-8555, Japan

M. Ishikawa

Kashiwa Kousei Clinic, Kashiwa, Chiba 277-0841, Japan the Mt5178A genotype group than in the Mt5178C group $(p=0.015)$.

Conclusions The Mt5178 C/A genotype may modify longitudinal changes in serum TC and HDLC levels in middle-aged Japanese men.

Keywords Mitochondrial polymorphism - Low-density lipoprotein cholesterol $\cdot$ High-density lipoprotein cholesterol · Follow-up study

\section{Introduction}

The mitochondrial DNA 5178 cytosine/adenine (Mt5178 C/A) polymorphism results in an amino acid polymorphism in NADH dehydrogenase subunit-2 237 leucine/methionine (ND2-237 Leu/Met) and is known as a longevity-associated polymorphism. Tanaka et al. [1, 2] suggested that the Mt5178 A genotype of the Mt5178 C/A polymorphism is associated with longevity in Japanese people. Specifically, they reported that the frequency of Mt5178A is significantly higher in centenarians than in the general population. The Mt5178A genotype is generally rare worldwide $[3,4]$, but it's frequency has been reported to be as high as $40-45 \%$ in the Japanese populace $[1,5-13]$. There have been a number of reports regarding an association between the Mt5178 C/A polymorphism and adult-onset diseases $[1,8,9,14]$, and individuals with Mt5178A appear to be resistant to atherosclerotic diseases.

Japanese individuals with the Mt5178C polymorphism are more susceptible to lifestyle-related atherosclerotic diseases, myocardial infarction [8,14], and cerebrovascular disorders [9] than those with Mt5178A. There is also evidence of differences in serum lipid levels being related to the Mt5178 C/A genotype [13, 15, 16]. In one study, the 
concentration of high-density lipoprotein cholesterol (HDLC) was significantly higher in males carrying the Mt5178A genotype than in males carrying the Mt5178C genotype; in the same study, triglyceride (TG) levels were significantly lower in females carrying the Mt5178A genotype than in those carrying the Mt5178C genotype [14].

The anti-atherogenic effects of the Mt5178A genotype may facilitate longevity in individuals with the Mt5178A polymorphism compared to those with Mt5178C. Based on previous findings that individuals with the Mt5178C genotype are more susceptible to arteriosclerosis diseases than those with the Mt5178A genotype, we examined changes in serum cholesterol levels between individuals with the Mt5178C genotype and those with the Mt5178A genotype over the course of 4 or 5 years because these lipid levels influence the development of arteriosclerosis.

\section{Materials and methods}

\section{Subjects}

Male volunteers $(n=449)$ were initially recruited from individuals who visited the Mito Red Cross Hospital for medical checkups between July 1999 and June 2000. Of the initial 449 recruits, 118 males who visited the hospital for medical checkups again between September 2003 and January 2005 and who were between 40 and 69 years of age at the second checkup were eligible for enrolment in the study. There were no prescribed interventions in diet, exercise, smoking, or drinking between the two medical checkups. Women were excluded from the study because there were not enough female recruits. The study was performed in accordance with the Declaration of Helsinki and approved by the Ethical Committee of Kyorin University. Written informed consent was obtained from volunteers before participation. The final study cohort comprised 110 Japanese men with a mean age of $52.3 \pm 6.0$ years [mean \pm standard deviation (SD)]. None of the subjects were taking dyslipidemic medications at the time of either medical checkup reported in this study.

\section{Clinical characteristics of subjects}

Blood samples were obtained after a minimum of a 12-h fasting period. Serum indicators were determined as described previously [13]. Briefly, serum concentrations of total cholesterol (TC) were measured on a Determiner L TC II apparatus (Kyowa Medex, Tokyo, Japan). Serum cholesterol concentrations were analyzed by routine methods used at Mito Red Cross Hospital, and serum concentrations of HDLC were measured with a Determiner
L HDL-C apparatus (Kyowa Medex). Low-density lipoprotein cholesterol (LDLC) concentrations were calculated according to Friedewald's formula after excluding the subjects whose TG levels were $>400 \mathrm{mg} / \mathrm{dl}$. The body mass index (BMI) was calculated as weight $(\mathrm{kg})$ divided by height squared $\left(\mathrm{m}^{2}\right)$. Information on the drinking and smoking habits of each subject was obtained from a selfadministrated questionnaire.

Normal ranges were defined as a TC of $<220 \mathrm{mg} / \mathrm{dl}$, LDLC of $<140 \mathrm{mg} / \mathrm{dl}$, and HDLC of $\geq 40 \mathrm{mg} / \mathrm{dl}$.

\section{Genotyping}

DNA was isolated from white blood cells and subjected to PCR-restriction fragment length polymorphism analysis using the forward PCR primer (5'-CTTAGCATACTCCTC AATTACCC- $\left.3^{\prime}\right)$, the reverse PCR primer $\left(5^{\prime}\right.$-CTGAATTC TTCGATAATGGCCCA- $3^{\prime}$ ), and the restriction enzyme $A l u \mathrm{I}$. PCR products lacking the $A l u \mathrm{I}$ site were designated as Mt5178A, and those possessing the AluI site were designated as Mt5178 C.

Statistical analysis

The changes in TC, LDLC, and HDLC levels were calculated by subtraction based on the measurements taken during the repeated visits, and the percentage of subjects outside the normal cholesterol levels between the two visits were compared by the chi-square test. Cholesterol levels in 1999-2000 and 2003-2004, respectively. in the whole cohort and each Mt5178 group were compared by the Wilcoxon signed-rank test. Changes in cholesterol levels over time between individuals with genotype Mt5178C and those with genotype Mt5178A type were compared by analysis of covariance adjusted for age, BMI, smoking and drinking habits.

The statistical analysis was performed with the Statistical Package for the Biosciences (SPBS v9.5; [17]). $p$ values of $<0.05$ were considered to be statistically significant.

\section{Results}

There were no significant differences between the subjects with the Mt5178C and Mt5178A genotypes in terms of the follow-up period, systolic blood pressure, diastolic blood pressure, fasting blood sugar. The average follow-up period was 4 years and 5 months (range 3 years, 7 months to 5 years, 3 months).

Age, BMI, smoking, and drinking habits in 1999-2000 and 2003-2004 are shown separately by genotype in Tables 1 and 2, respectively. Among our study subjects, 
Table 1 Characteristics of study subjects according to mitochondrial DNA 5178 cytosine/adenine (Mt5178 C/A) genotype based on the 1999-2000 checkups

Data are presented as the mean \pm standard deviation, unless indicated otherwise

$B M I$ Body mass index, $S B P$ systolic blood pressure, $D B P$ diastolic blood pressure

\begin{tabular}{llll}
\hline Patient characteristics & Mt5178 C $(n=64)$ & Mt5178 A $(n=46)$ & Total $(n=110)$ \\
\hline Age (years) & $53.0 \pm 6.2$ & $51.4 \pm 5.8$ & $52.3 \pm 6.0$ \\
BMI $\left(\mathrm{kg} / \mathrm{m}^{2}\right)$ & $23.3 \pm 2.4$ & $24.0 \pm 2.5$ & $23.6 \pm 2.4$ \\
SBP $(\mathrm{mmHg})$ & $127.0 \pm 16.0$ & $122.5 \pm 12.1$ & $125.1 \pm 14.6$ \\
DBP $(\mathrm{mmHg})$ & $74.0 \pm 9.6$ & $71.5 \pm 7.9$ & $73.0 \pm 9.0$ \\
Fasting blood sugar $(\mathrm{mg} / \mathrm{dl})$ & $101.4 \pm 27.5$ & $103.6 \pm 21.5$ & $102.3 \pm 25.1$ \\
Smoking habit & & & \\
$\quad$ Current smokers & $28(43.8 \%)$ & $21(45.7)$ & $39(44.5)$ \\
$\quad$ Past smokers & $23(35.9)$ & $15(32.6)$ & $21(19.1)$ \\
$\quad$ Never smoked & $11(17.2)$ & $10(21.7)$ & $38(34.5)$ \\
Frequency of alcohol intake $(n)$ & & $17(37.0)$ & $32(29.1)$ \\
$\quad$ Every day & $21(32.8)$ & $11(23.9)$ & $20(18.2)$ \\
$\quad$ Several/week & $21(32.8)$ & $9(19.6)$ & $20(18.2)$ \\
$\quad$ Several/month & $11(17.2)$ & $9(19.6)$ & \\
$\quad$ No alcohol & $11(17.2)$ & & \\
\hline
\end{tabular}

Table 2 Characteristics of study subjects by Mt5178 C/A genotype from the 2003-2004 checkups

\begin{tabular}{|c|c|c|c|}
\hline $\begin{array}{l}\text { Patient } \\
\text { characteristics }\end{array}$ & $\begin{array}{l}\text { Mt5178 C } \\
(n=64)\end{array}$ & $\begin{array}{l}\text { Mt5178 A } \\
(n=46)\end{array}$ & $\begin{array}{l}\text { Total } \\
(n=110)\end{array}$ \\
\hline Age (years) & $57.4 \pm 6.2$ & $55.8 \pm 5.6$ & $56.8 \pm 6.0$ \\
\hline BMI $\left(\mathrm{kg} / \mathrm{m}^{2}\right)$ & $23.6 \pm 2.6$ & $24.0 \pm 2.5$ & $23.8 \pm 2.5$ \\
\hline $\mathrm{SBP}(\mathrm{mmHg})$ & $122.2 \pm 11.8$ & $116.6 \pm 10.5$ & $119.9 \pm 11.6$ \\
\hline DBP (mmHg) & $77.2 \pm 8.7$ & $75.2 \pm 7.7$ & $76.3 \pm 8.3$ \\
\hline $\begin{array}{l}\text { Fasting blood sugar } \\
\quad(\mathrm{mg} / \mathrm{dl})\end{array}$ & $104.3 \pm 15.3$ & $110.7 \pm 28.7$ & $106.9 \pm 22.0$ \\
\hline \multicolumn{4}{|l|}{ Smoking habit $(n)$} \\
\hline Current smokers & $23(35.9 \%)$ & $16(34.8)$ & $39(35.5)$ \\
\hline Past smokers & $28(43.8)$ & $20(43.5)$ & $48(43.6)$ \\
\hline Never smokers & $11(17.2)$ & $10(21.7)$ & $22(20.0)$ \\
\hline \multicolumn{4}{|c|}{ Frequency of alcohol intake $(n)$} \\
\hline Every day & $21(34.4)$ & $18(40.0)$ & $39(36.8)$ \\
\hline Several/week & $17(27.9)$ & $12(26.7)$ & $29(27.4)$ \\
\hline Several/month & $11(18.0)$ & $5(11.1)$ & $16(15.1)$ \\
\hline No alcohol & $12(19.7)$ & $10(22.2)$ & $22(20.8)$ \\
\hline
\end{tabular}

Data are presented as the mean \pm standard deviation, unless indicated otherwise

the relative frequency of Mt5178C and Mt5178A was 58.2 (64/110) and 41.8\% (46/110), respectively. There were no statistically significant differences between the Mt5178 C/A genotypes in terms of age, BMI, or smoking and drinking habits, and no significant change was seen between 1999-2000 and 2003-2004 in BMI, smoking habits, or frequency of alcohol intake in either group.

Table 3 shows the prevalence of subjects with serum cholesterol levels falling within and outside the normal range at the time of both medical checkup visits. The percentage of subjects whose TC fell outside the normal range was $30.9 \%(34 / 110)$ in $1999-2000$ and $17.3 \%$ (19/ $110)$ in 2003-2004 ( $p=0.027$, chi-square test).

In the Mt5178C group, the percentage of subjects with a TC level that fell outside of the normal range did not change significantly between 1999-2000 and 2003-2004, while in the Mt5178A group, this decreased from $37.0 \%$ (17/46) in $1999-2000$ to $13.0 \%(6 / 46)$ in 2003-2004 ( $p=0.016)$. No significant changes were observed between the two checkups in these percentages for the LDLC and HDLC levels in whole cohort or in either genotype group.

The means and SD of the TC, LDLC, and HDLC levels in each period, 1999-2000 and 2003-2004, and the change by genotype are shown in Table 4 . The mean TC level decreased significantly $(p=0.020)$ in the whole cohort. Although the TC level in the Mt5178C group decreased from 1999-2000 to 2003-2004, this decrease was not statistically significant. In contrast, the decrease in TC levels in the Mt5178A group was significant $(p=0.016)$. No significant difference was observed in the change between the Mt5178C $(-3 \mathrm{mg} / \mathrm{dl})$ and Mt5178A $(-9.5 \mathrm{mg} / \mathrm{dl})$ groups $(p=0.269)$.

The LDLC level decreased slightly from 1999-2000 to 2003-2004 in the whole cohort and in both the Mt5178C and Mt5178A groups when considered separately, but these decreases over time were not significant, and the changes in LDLC levels were also not significantly different between groups (whole cohort, A genotype, and $\mathrm{C}$ genotype).

The HDLC level in the Mt5178A group significantly increased from 1999-2000 to 2003-2004 $(p=0.018)$, while the HDLC level in the Mt5178C group remained unchanged. The change in the Mt5178C $(0 \mathrm{mg} / \mathrm{dl})$ and the Mt5178A (3 mg/dl) groups was significantly different $(p=0.015)$.

In the univariate analysis of TC, age, BMI, drinking frequency, and smoking habit were significant at $p<0.05$; 
Table 3 Proportion of subjects with serum cholesterol levels falling within and outside the normal range at the 1999-2000 and 2003-2004 checkups

\begin{tabular}{|c|c|c|c|c|}
\hline \multirow{2}{*}{$\begin{array}{l}\text { Serum cholesterol } \\
\text { components }\end{array}$} & \multicolumn{2}{|l|}{ 1999-2000 } & \multicolumn{2}{|l|}{ 2003-2004 } \\
\hline & $\begin{array}{l}\text { Within } \\
\text { normal } \\
\text { range }(\%)\end{array}$ & $\begin{array}{l}\text { Outside } \\
\text { normal } \\
\text { range }(\%)\end{array}$ & $\begin{array}{l}\text { Within } \\
\text { normal } \\
\text { range }(\%)\end{array}$ & $\begin{array}{l}\text { Outside } \\
\text { normal } \\
\text { range }(\%)\end{array}$ \\
\hline \multicolumn{5}{|l|}{ Total } \\
\hline $\mathrm{TC}$ & $76(69.1)$ & $34(30.9)$ & $91(82.7)$ & $19(17.3)^{*}$ \\
\hline LDLC & 77 (72.6) & $29(27.4)$ & $85(80.2)$ & $21(19.8)$ \\
\hline HDLC & $98(89.1)$ & $12(10.9)$ & $97(88.2)$ & 13 (11.8) \\
\hline \multicolumn{5}{|l|}{$\mathrm{C}$} \\
\hline $\mathrm{TC}$ & 47 (73.4) & $17(26.6)$ & $51(79.7)$ & $13(20.3)$ \\
\hline LDLC & $46(74.2)$ & $16(25.8)$ & $49(79.0)$ & $13(21.0)$ \\
\hline HDLC & $56(87.5)$ & $8(12.5)$ & $56(87.5)$ & $8(12.5)$ \\
\hline \multicolumn{5}{|l|}{ A } \\
\hline $\mathrm{TC}$ & $29(63.0)$ & $17(37.0)$ & $40(87.0)$ & $6(13.0)^{*}$ \\
\hline LDLC & $31(70.5)$ & $13(29.5)$ & $36(81.8)$ & $8(18.2)$ \\
\hline HDLC & $42(91.3)$ & $4(8.7)$ & $41(89.1)$ & $5(10.9)$ \\
\hline
\end{tabular}

$* p<0.05$, compared to the ratio from the 1999-2000 checkup by Chi-square test

TC Total cholesterol, LDLC low-density lipoprotein cholesterol, $H D L C$ high-density lipoprotein cholesterol

Data are presented as the number of subjects, with the percentage given in parenthesis

in the univariate analysis of HDLC and LDLC, age, BMI, and drinking frequency were significant at $p<0.05$. Although smoking habit was not significant in the univariate analyses of HDLC and LDLC, it is clear that smoking influences HDLC and LDLC. Therefore, smoking habit was also used as the variable in the covariance analyses of HDLC and LDLC.

\section{Discussion}

In this investigation, the decrease in TC levels in the Mt5178A group was significant after age, BMI, smoking, and drinking habits had been accounted for, although no significant difference was observed in the change between the Mt5178C and Mt5178A groups. HDLC levels were found to have significantly improved in the Mt5178A group during the 4 or 5 years between the checkups, whereas no significant changes were observed between checkups in the Mt5178C group. The frequency of the Mt5178A genotype observed among our subject cohort was similar to the $43.8 \%$ reported by Tanaka et al. [1], thus suggesting that there is no genetic bias in the subjects in this study.

The general assumption is that in both men and women, in the absence of intervention, the serum HDLC level will remain relatively stable throughout adult life [18-20] or to show a tendency to decrease as a person ages [18, 21-23]. In their study, Wilson et al. [18] showed that HDLC decreased in all age groups of men and women (range at baseline 20-79 years) over follow-up intervals of 8 years and that these changes occurred among healthy elderly individuals not taking lipid-altering medications. They also reported that age itself was an important determinant of change in HDLC levels. The strength of the relationship between HDLC and BMI was weaker in men older than 45 years than in men at younger ages, whereas the decline in HDLC was proportional to change in BMI among men of all ages. Furthermore, cigarette smoking and alcohol intake had less influence on HDLC levels in people over the age of 50 years than in younger individuals; nevertheless, the cessation of either cigarette smoking or high alcohol intake was associated with increased HDLC in both men and women [18]. In our study, there were no significant changes in the factors (specifically LDLC level, weight, BMI, or smoking and drinking habits) known to affect HDLC levels in any of the three groups (whole cohort, Mt5178A group, or Mt5178C group) between the first and follow-up checkups; therefore, we suggest that the Mt5178A variant itself was responsible for the observed increase in HDLC levels in the Mt5178A group.

Although the mechanism by which Mt5178A influences the HDLC levels is not clear, the substitution of adenine by cytosine at Mt5178 results in a deduced amino acid change from leucine to methionine, and this change at residue 237 of NADH dehydrogenase subunit 2 may bring the functional change in NADH dehydrogenase. Levine et al. [24] demonstrated that a variety of oxidants react readily with methionine to form methionine sulfoxide and that surfaceexposed methionine residues create a high concentration of reactant, available as an efficient oxidant scavenger. Reduction back to methionine by methionine sulfoxide reductases would allow the antioxidant system to function catalytically. Thus, methionine may scavenge many oxidizing molecules [24, 25]. Methionine in the proteins of the Mt5178A group could be considered to be more efficient in defending against oxidative stress and keeping serum lipid levels normal in comparison to those of the Mt5178C group. Gusdon et al. [26] examined mitochondrial reactive oxygen species (ROS) production in mice with the typical Mt4738C and Mt4738A genotype, respectively, which encoded in a leucine to methionine amino acid substitution at NADH dehydrogenase subunit 2 gene. These authors showed that Mt4738A mice exhibited lower ROS production. Thus, the single similar nucleotide polymorphism in Mt5178C/A may itself be involved in the functional change against ROSassociated conditions alike. Further investigation to identify the biochemical mechanism in experimental animals will be necessary as well as human epidemiological studies. 
Table 4 Total cholesterol, high-density lipoprotein cholesterol and low-density lipoprotein cholesterol among the study subjects in 1999-2000 and 2003-2004 years and changes

\begin{tabular}{|c|c|c|c|}
\hline Serum cholesterol components ${ }^{\mathrm{a}}$ & $1999-2000^{\mathrm{b}}$ & $2003-2004^{\mathrm{b}}$ & Changes $^{c}$ \\
\hline \multicolumn{4}{|l|}{ Total group } \\
\hline $\mathrm{TC}(n=110)$ & $203.1 \pm 35.0$ & $197.1 \pm 28.0^{*}$ & $-7.5(-73$ to 64$)$ \\
\hline $\operatorname{LDLC}(n=106)$ & $122.7 \pm 31.0$ & $119.9 \pm 26.5$ & $-3(-65$ to 70$)$ \\
\hline $\operatorname{HDLC}(n=110)$ & $53.5 \pm 11.6$ & $54.2 \pm 11.6$ & $1(-19$ to 28$)$ \\
\hline \multicolumn{4}{|l|}{ Mt5178 C group } \\
\hline $\mathrm{TC}(n=64)$ & $202.5 \pm 36.2$ & $199.0 \pm 29.9$ & $-3(-73$ to 64$)$ \\
\hline $\operatorname{LDLC}(n=62)$ & $122.7 \pm 32.1$ & $121.6 \pm 28.1$ & $-3(-65$ to 70$)$ \\
\hline $\operatorname{HDLC}(n=64)$ & $54.7 \pm 11.7$ & $54.0 \pm 10.8$ & $0(-19$ to 15$)$ \\
\hline \multicolumn{4}{|l|}{ Mt5178 A group } \\
\hline $\mathrm{TC}(n=46)$ & $204.0 \pm 33.6$ & $194.5 \pm 25.2^{*}$ & $-9.5(-71$ to 45$)$ \\
\hline $\operatorname{LDLC}(n=44)$ & $122.8 \pm 29.8$ & $117.5 \pm 24.2$ & $-4(-53$ to 53$)$ \\
\hline $\operatorname{HDLC}(n=46)$ & $51.8 \pm 11.3$ & $54.5 \pm 12.6^{*}$ & $3(-10 \text { to } 28)^{\#}$ \\
\hline \multicolumn{4}{|c|}{$\begin{array}{l}{ }^{*} p<0.05 \text {, compared to the level of } 1999-2000 \text { by Wilcoxon signed-rank test; }{ }^{\#} p<0.05 \text {, compared to the C genotype and A genotype by } \\
\text { analysis of covariance adjusted for age, BMI, smoking habit, and drinking habit }\end{array}$} \\
\hline
\end{tabular}

Individuals with Mt5178A have been reported to be relatively less susceptible to adult-onset diseases, such as myocardial infarction and atherosclerosis $[8,14]$. Based on the results of a study carried out by Gordon et al. in men between the ages of 30 and 69 years [27], a $1 \mathrm{mg} / \mathrm{dl}$ increment in HDLC was associated with a significant $1.9-2.3 \%$ decrement in the incidence rates of coronary heart disease (CHD) and with a 3.7-4.7\% decrement in CHD mortality rates. It would also appear that a $2.7 \mathrm{mg} / \mathrm{dl}$ increment in HDLC in individuals with the Mt5178A genotype influences the incidence and mortality rates of CHD. In another study involving Japanese men aged $\geq 30$ years, there was a positive graded relationship between TC $(>160 \mathrm{mg} / \mathrm{dl})$ and mortality due to coronary artery disease after adjustment for age, serum albumin, BMI, hypertension, diabetes, cigarette smoking, and alcohol intake [28]. On the grounds that the TC group even within normal ranges had a gradual higher multivariate-adjusted hazard ratio for CHD mortality [28], these authors proposed that slight decreases of TC shown in the Mt5178A subjects could influence the risk of CHD incidence.

Brousseau et al. [29] reported that a significant increase in the frequency of the mutant G3456C allele of the ABCA1 gene was observed in the Veterans Affairs Cooperative HDL Cholesterol Intervention Trial (VA-HIT), which compared men with a history of CHD and a low serum HDLC level $(\leq 40 \mathrm{mg} / \mathrm{dl})$ to another group of men without any history of CHD. These authors reported that the G3456C variant was associated with a more than twofold increase in risk for CHD end points, defined as death due to
CHD or nonfatal myocardial infarction, whereas no significant difference was observed in the HDLC level between those individuals with the ABC-A1 genotype in the VA-HIT [29]. Taking such research into consideration, the question of whether the Mt5178 C/A polymorphism influences the risk factors of CHD, including high blood pressure and blood sugar levels as well as serum lipids, also warrants further long-term follow-up studies.

In this study, the HDLC level in individuals with the Mt5178A genotype significantly increased from 1999-2000 to 2003-2004, while that in individuals with the Mt5178C genotype was unchanged; there were also no significant differences observed in the HDLC levels between the two groups (Mt5178C and Mt5178A genotypes) in the two periods. One of the limitations of this study relates to the generalizability of the results. The short interval period evaluated is a potential weakness of this paper, as is the small sample size. The reason why individuals with the Mt5178A polymorphism had a lower level of HDLC than those with Mt5178C at baseline is unknown and requires a large-scale study for further elucidation. As the subjects in our study were all Japanese and only a limited number of measurements was available, the subjects should have been recruited from at least two or more areas in Japan. Furthermore, a larger cohort study involving different ethnic groups is needed to definitively demonstrate the contributions of the Mt 5178 C/A polymorphism to serum cholesterol levels. In our research, dietary habit and physical activity were not added to the confounding variables under consideration. As both dietary habit and physical activity 
influence cholesterol levels, an analysis in which these variables are considered is required. Moreover, for practical reasons in the routine health checkup setting, LDLC was estimated using the values of $\mathrm{TC}$ and $\mathrm{TG}$ based on Friedewald's formula. However, the TG values can readily fluctuate, depending on the number of fasting hours and the last dietary intake; thus, direct, repeated measurements are required to evaluate LDLC as precisely as possible.

In conclusion, among our study cohort of middle-aged Japanese men, the Mt5178 C/A polymorphism tended to contribute to longitudinal changes in TC and HDLC. Our findings may suggest the possibility of a more personalized, patient-specific preventative intervention based on genotyping. They may also suggest that individuals with the Mt5178C genotype should receive more careful healthmaintenance instructions during the course of preventive consultations for atherogenic diseases.

Acknowledgments The authors would like to thank Naomi Matsunaga and Yoshiko Abe of the Kyorin University School of Medicine for their continuous support to this study.

Conflict of interest None.

\section{References}

1. Tanaka M, Gong JS, Zhang J, Yoneda M, Yagi K. Mitochondrial genotype associated with longevity. Lancet. 1998;351(9097): 185-6.

2. Tanaka M, Gong J, Zhang J, Yamada Y, Borgeld HJ, Yagi K. Mitochondrial genotype associated with longevity and its inhibitory effect on mutagenesis. Mech Ageing Dev. 2000;116(2-3): 65-76.

3. Cann RL, Stoneking M, Wilson AC. Mitochondrial DNA and human evolution. Nature. 1987;325(6099):31-6.

4. Yao YG, Kong QP, Zhang YP. Mitochondrial DNA 5178A polymorphism and longevity. Hum Genet. 2002;111(4-5):462-3.

5. Shimokata H, Yamada Y, Nakagawa M, Okubo R, Saido T, Funakoshi A, et al. Distribution of geriatric disease-related genotypes in the National Institute for Longevity Sciences, Longitudinal Study of Aging (NILS-LSA). J Epidemiol. 2000; 10[Suppl 1]:S46-55.

6. Wang D, Taniyama M, Suzuki Y, Katagiri T, Ban Y. Association of the mitochondrial DNA 5178A/C polymorphism with maternal inheritance and onset of type 2 diabetes in Japanese patients. Exp Clin Endocrinol Diabetes. 2001;109(7):361-4.

7. Matsunaga H, Tanaka Y, Tanaka M, Gong JS, Zhang J, Nomiyama T, et al. Antiatherogenic mitochondrial genotype in patients with type 2 diabetes. Diabetes Care. 2001;24(3):500-3.

8. Mukae S, Aoki S, Itoh S, Sato R, Nishio K, Iwata T, et al. Mitochondrial 5178A/C genotype is associated with acute myocardial infarction. Circ J. 2003;67(1):16-20.

9. Ohkubo R, Nakagawa M, Ikeda K, Kodama T, Arimura K, Akiba $\mathrm{S}$, et al. Cerebrovascular disorders and genetic polymorphisms: mitochondrial DNA5178C is predominant in cerebrovascular disorders. J Neurol Sci. 2002;198(1-2):31-5.

10. Aoyama M, Shidoji Y, Saimei M, Tsunawake N, Ichinose M. Phenotypic linkage between single-nucleotide polymorphisms of beta3-adrenergic receptor gene and NADH dehydrogenase subunit-2 gene, with special reference to eating behavior. Biochem Biophys Res Commun. 2003;309(1):261-5.

11. Kato T, Kunugi H, Nanko S, Kato N. Association of bipolar disorder with the 5178 polymorphism in mitochondrial DNA. Am J Med Genet. 2000;96(2):182-6.

12. Iwao N, Iwao S, Kobayashi F, Tajima K, Tanaka M, Atsuta Y, et al. No association of the mitochondrial genotype (Mt5178A/C) with six cancers in a Japanese population. Asian Pac J Cancer Prev. 2003;4(4):331-6.

13. Kokaze A, Ishikawa M, Matsunaga N, Yoshida M, Sekine Y, Teruya K, et al. Association of the mitochondrial DNA 5178 A/C polymorphism with serum lipid levels in the Japanese population. Hum Genet. 2001;109(5):521-5.

14. Takagi K, Yamada Y, Gong JS, Sone T, Yokota M, Tanaka M. Association of a $5178 \mathrm{C}->$ A (Leu237Met) polymorphism in the mitochondrial DNA with a low prevalence of myocardial infarction in Japanese individuals. Atherosclerosis. 2004;175(2): 281-6.

15. Kokaze A, Ishikawa M, Matsunaga N, Yoshida M, Sekine Y, Sekiguchi K, et al. Longevity-associated mitochondrial DNA $5178 \mathrm{~A} / \mathrm{C}$ polymorphism modulates effects of daily drinking and cigarette consumption on serum triglyceride levels in middleaged Japanese men. Exp Gerontol. 2003;38(10):1071-6.

16. Kokaze A, Ishikawa M, Matsunaga N, Yoshida M, Makita R, Satoh M, et al. Overview of longevity-associated mitochondrial DNA $5178 \mathrm{C} / \mathrm{A}$ polymorphism and a discussion of its modulation of the effects of habitual smoking on serum total and LDL cholesterol levels in middle-aged Japanese men. In: Woods CR, editor. Trends in DNA research. New York: Nova Science; 2006. p. $33-51$.

17. Murata K, Yano E. Medical statistics for evidence-based medicine with SPBS user's guide. Tokyo: Nankodo Publisher; 2002.

18. Wilson PW, Anderson KM, Harris T, Kannel WB, Castelli WP. Determinants of change in total cholesterol and HDL-C with age: the Framingham Study. J Gerontol. 1994;49(6):M252-7.

19. Wallace RB, Colsher PL. Blood lipid distributions in older persons. Prevalence and correlates of hyperlipidemia. Ann Epidemiol. 1992;2(1-2):15-21.

20. Frishman WH, Ooi WL, Derman MP, Eder HA, Gidez LI, Ben-Zeev D, et al. Serum lipids and lipoproteins in advanced age. Intraindividual changes. Ann Epidemiol. 1992;2(1-2):43-50.

21. Rahilly-Tierney C, Bowman TS, Djoussé L, Sesso HD, Gaziano JM. Change in high-density lipoprotein cholesterol and incident coronary heart disease in apparently healthy male physicians. Am J Cardiol. 2008;102(12):1663-7.

22. Derby CA, Feldman HA, Bausserman LL, Parker DR, Gans KM, Carleton RA. HDL cholesterol: trends in two southeastern New England communities, 1981-1993. Ann Epidemiol. 1998;8(2): 84-91.

23. Sprafka JM, Burke GL, Folsom AR, Luepker RV, Blackburn H. Continued decline in cardiovascular disease risk factors: results of the Minnesota Heart Survey, 1980-1982 and 1985-1987. Am J Epidemiol. 1990;132(3):489-500.

24. Levine RL, Mosoni L, Berlett BS, Stadtman ER. Methionine residues as endogenous antioxidants in proteins. Proc Natl Acad Sci USA. 1996;93(26):15036-40.

25. Luo S, Levine RL. Methionine in proteins defends against oxidative stress. FASEB J. 2009;23(2):464-72.

26. Gusdon AM, Votyakova TV, Mathews CE. mt-Nd2 suppresses reactive oxygen species production by mitochondrial complexes I and III. J Biol Chem. 2008;283(16):10690-7.

27. Gordon DJ, Probstfield JL, Garrison RJ, Neaton JD, Castelli WP, Knoke JD, et al. High-density lipoprotein cholesterol and cardiovascular disease. Four prospective American studies. Circulation. 1989;79(1):8-15. 
28. Okamura T. Dyslipidemia and cardiovascular disease: a series of epidemiologic studies in Japanese populations. J Epidemiol. 2010;20(4):259-65.

29. Brousseau ME, Bodzioch M, Schaefer EJ, Goldkamp AL, Kielar $\mathrm{D}$, Probst $\mathrm{M}$, et al. Common variants in the gene encoding
ATP-binding cassette transporter 1 in men with low HDL cholesterol levels and coronary heart disease. Atherosclerosis. 2001;154(3):607-11. 